\title{
Optimal Driving Range for Battery Electric Vehicles Based on Modeling Users' Driving and Charging Behavior
}

\author{
Zhenbo Lu, ${ }^{1}$ Qi Zhang $\mathbb{D}^{2}{ }^{2}$ Yu Yuan, ${ }^{2}$ and Weiping Tong ${ }^{2}$ \\ ${ }^{1}$ Intelligent Transportation System Research Center, Southeast University, Nanjing, China \\ ${ }^{2}$ Jiangsu Key Laboratory of Urban ITS, Jiangsu Province Collaborative Innovation Center of Modern Urban Traffic Technologies, \\ School of Transportation, Southeast University, Nanjing, China \\ Correspondence should be addressed to Qi Zhang; zhangqi_1008@seu.edu.cn
}

Received 25 April 2020; Revised 20 May 2020; Accepted 3 June 2020; Published 16 June 2020

Academic Editor: Weiwei Qi

Copyright (C) 2020 Zhenbo Lu et al. This is an open access article distributed under the Creative Commons Attribution License, which permits unrestricted use, distribution, and reproduction in any medium, provided the original work is properly cited.

\begin{abstract}
This paper proposes a simulation approach for the optimal driving range of battery electric vehicles (BEVs) by modeling the driving and charging behavior. The driving and charging patterns of BEV users are characterized by reconstructing the daily travel chain based on the practical data collected from Shanghai, China. Meanwhile, interdependent behavioral variables for daily trips and each trip are defined in the daily trip chain. To meet the goal of the fitness of driving range, a stochastic simulation framework is established by the Monte Carlo method. Finally, with consideration of user heterogeneity, the optimal driving range under different charging scenarios is analyzed. The findings include the following. (1) The daily trip chain can be reconstructed through the behavioral variables for daily trips and each trip, and there is a correlation between the variables examined by the copula function. (2) Users with different daily travel demand have a different optimal driving range. When choosing a BEV, users are recommended to consider that the daily vehicle kilometers traveled are less than $34 \%$ of the battery driving range. (3) Increasing the charging opportunity and charging power is more beneficial to drivers who are characterized by high daily travel demand. (4) On the premise of meeting travel demand, the beneficial effects of increased fast-charging power will gradually decline.
\end{abstract}

\section{Introduction}

Battery electric vehicles (BEVs) have the outstanding advantages in zero tailpipe emissions, low noise, convenient maintenance, and high energy conversion efficiency. The deployment of BEVs helps to reduce oil dependence, improve air quality, and reduce pollutions and greenhouse gas emissions [1]. Promoting the development of BEVs is considered as one of the promising solutions for the treatment of severe air pollution in metropolises [2]. The incentives, such as subsidies and tax credits, have effectively promoted the public acceptance for switching to BEVs. For instance, in many mega cities in China, like Beijing, Shanghai, and Hangzhou, the number of vehicle license plates issued per month (car ownership) is under strict control, and the local government has also launched the free licensing policies for BEVs [3].

However, due to the limited battery capacity and charging facilities, inconvenient charging is still an important obstacle to the promotion of BEVs [4]. Compared to the conventional internal combustion engine vehicles (ICEV), BEVs have a shorter driving range, generally $150 \mathrm{~km}-400 \mathrm{~km}$. Meanwhile, it usually takes hours to charge $[5,6]$. Potential customers have repeatedly been found to prefer vehicles with considerably higher available range because of the range anxiety [7]. Although the long driving range design helps to alleviate the user's range anxiety, it results in a higher expenditure on purchase and simultaneously, the affordability and cost-effectiveness is lowered [8]. Optimizing driving range of BEVs based on users' daily travel demand is one of the feasible ways to solve this problem, and it is also the direction of breakthrough for this paper.

The main contribution of this paper is optimizing the driving range for different types of $\mathrm{BEV}$ users based on the real-world BEV usage data, specifically including the following: (1) constructing the daily trip chain for BEV drivers 
by combining behavioral variables for daily trip and each trip; (2) applying the copula function to examine the dependence between daily travel variables; (3) proposing a simulation framework for the Mote Carlo method to simulate the daily trip chain; and (4) quantifying the fitness of driving range to find the optimal driving range for different drivers.

The remainder of the paper is organized as follows: after a review of relevant literature, Section 3 defines the research problem, followed by the stochastic formulation of driver behavioral models and the procedure of the Monte Carlo simulation in Section 4. Results and discussion are presented in Section 5, and Section 6 concludes this study.

\section{Literature Review}

Researchers have conducted a series of studies on the travel and charging behaviors of users based on the trajectory data of new energy vehicles (NEVs). In order to understand travel and charging behaviors, many existing studies focused on the distribution of distance traveled per day $[5,9,10]$, the start time of charging [11-13], daily vehicle kilometers traveled (DVKT) $[10,12,14]$, distance traveled between consecutive charges $[5,15,16]$, and battery state of charge (SOC) before and after charging $[11,17,18]$. For example, $\mathrm{Wu}$ et al. [9] collected the travel data of 403 plug-in hybrid cars and analyzed the effects of the daily mileage and the mileage between the two charges. Pearre et al. [5] analyzed 470 vehicles with more than 50 days' travel data in Atlanta, USA, and found that the highest probability of DVKT distribution was $19.2-25.6 \mathrm{~km}$; a wide variation in driving distances was observed from different drivers.

Based on the driving behaviors of ICEV and NEV users, how to optimize battery capacity or driving range of electric vehicles (EVs) is studied. Li et al. [19] proposed a hybrid distribution model to describe the daily travel mileage for the purpose of optimizing battery capacity; the testing results indicated that the mixture distribution model could satisfy various drivers. In addition, Dong and Lin [15] proposed the concept of BEV feasibility through a stochastic modeling approach to characterize BEV drivers' behavior. The range of comfort level of the drivers with different driving characteristics was studied to explore solutions to reduce range anxiety. However, although the driving patterns characterizing BEVs are stochastic, the researchers found that the variables are interdependent. After modeling the dependence structure between six variables using a nonparametric copula function, Brady and O'Mahony [20] applied a stochastic simulation methodology to generate a schedule of daily travel and charging profile.

The travel pattern of a particular BEV owner is relatively constant, yet the uncertainties make the travel demand vary from different drivers (i.e., interdriver heterogeneity). Using the data collected from 50 BEVs in Shanghai, China, Yang et al. [11] found the interdriver heterogeneity existing in driving and charging behaviors in terms of distance traveled per day, start time of charging, daily number of charging events, distance traveled between consecutive charges, SOC before and after charging, and time-of-day electricity demand. Due to the heterogeneities, it is unrealistic for vehicle manufacturers to make the driving range exactly equal to drivers' travel demand. The method of Monte Carlo simulation with the trip chain theory is often used to depict drivers' stochastic behaviors. For example, based on the survey data of National Household Travel Survey (NHTS), Jianfeng et al. [12] fitted the characteristics of the trip chain and analyzed the charging demand by the Monte Carlo simulation. Shuqiang et al. [21] proposed a method to analyze the charging demand of electric vehicles based on the trip chain theory; the Monte Carlo method was applied to explore the probability distribution characteristics of EVs parking time in different regions during one-day travel.

In summary, for the study of optimizing driving range, it is reasonable to consider the correlation between behavior variables and the heterogeneity of users in the modeling process. In addition, practical BEV usage data is rarely utilized to support users daily trip chain reconstruction based on travel and charging behavior. In view of the above methods and shortcomings, this paper proposes a probability distribution model based on the daily user trip chain by considering the correlation between the variables of daily trips and each trip. Combining different scenario settings, users with different driving behavior are analyzed for optimal driving range using Monte Carlo simulation method.

\section{Problem Statement}

3.1. Daily Trip Chain for BEV Users. The term of "trip chain" has different definitions [22]. To describe each individual's daily movement, we define the trip chain as a sequence of trips bounded by dwells. A trip that commutes between two anchor destinations, such as home to work, or work to home, is powered by electricity, and the dwell between two consecutive trips offers charging opportunities for BEVs.

Assuming BEV drivers leave home in the morning of the travel day with a full battery, the departure time of the first $\operatorname{trip} T_{0}$ is recorded. With the travel time of the first trip $t_{1}$ and the travel speed of the first trip $v_{1}$, the distance traveled and the energy consumed for the first trip are estimated. The departure time for the next trip is determined by the dwell time after the first trip $s_{1}$. Drivers may plug in their vehicles at the dwell place with considerations that the dwell time is long enough, the SOC is below a certain value (SOC before charging: $\mathrm{SOC}_{n}^{\prime}$ ), and the charging facility is available. After the total number of trips $N$ that day, the driver returned home, and daily vehicle kilometers traveled $D$ are recorded. The electric energy consumed by the $n$-th trip $E_{n}^{t}$ can be estimated by the travel time of the $n$-th trip $t_{n}$ and the average travel speed of the $n$-th trip $v_{n}$. The energy obtained from the $n$-th dwell $E_{n}^{c}$ is determined by the time length of the $n$-th dwell $s_{n}$ and the power of charging facility. Thus, the daily trip chain is formed by the abovementioned determinants, which include a variety of behavioral variables that describe daily trips and each trip. Table 1 summarizes the behavioral variables of the daily trip chain. The distributions of behavioral variables are derived from the BEV dataset collected from Shanghai, China, in the next section. Once the coverage of charging infrastructure is given, the fitness of 
TABLE 1: A summary of the behavioral variables of the daily trip chain.

\begin{tabular}{lc}
\hline $\begin{array}{l}\text { Variables for daily } \\
\text { trips }\end{array}$ & $\begin{array}{c}\text { Number of daily trips } N \\
\text { The departure time of the first trip } T_{0} \\
\text { Daily vehicle kilometers traveled } D \\
\text { The travel time of the } n \text {th trip } t_{n} \\
\text { The average travel speed of the } n \text {th trip } v_{n} \\
\text { Variables for each trip } \\
\text { Dwell time between two consecutive trips } \\
\text { Variables for charging }\end{array} \quad$ SOC before charging $\mathrm{SOC}_{n}^{\prime}$ \\
\hline
\end{tabular}

the driving range, indicating whether the BEV driving range is capable of covering the daily trip chain, is quantified.

3.2. Correlation between Behavioral Variables. The behavioral variables in the daily trip chain are mutually influential and not independently distributed. Studies have demonstrated the correlation between the variables for daily trips [20] (i.e., number of daily trips $N$, the departure time of the first trip $T_{0}$, and daily vehicle kilometers traveled $D$ ) and applied the copula function to combine the distributions between them. The copula function is called a "join function" or a "dependent function," which is a function that connects the joint distribution of multiple random variables with their respective marginal distributions [23]. Specifically, in accordance with Sklar's theorem [24], the continuous random variables $x_{1}, x_{2}, \ldots, x_{n}$ with cumulative distribution functions (CDFs) $F_{1}\left(x_{1}\right), F_{2}\left(x_{2}\right), \ldots, F_{n}\left(x_{n}\right)$, respectively, are joined by copula $C$ if their joint distribution function can be expressed as

$$
F\left(x_{1}, x_{2}, \ldots, x_{n}\right)=C\left(F_{1}\left(x_{1}\right), F_{2}\left(x_{2}\right), \ldots, F_{n}\left(x_{n}\right)\right) .
$$

Further, the three behavior variables of each trip not only affect each other, but also depend on the three variables of daily trip. Equation (2) establishes the link between each trip variable and the daily trip variable:

$$
D=\sum_{n}^{N} t_{n} \cdot v_{n}
$$

Daily vehicle kilometers traveled $D$ are the cumulative sum of the travel time of each trip $t_{n}$ multiplied by the average speed of each trip $v_{n}$ under the number of daily trips $N$.

3.3. Fitness of Driving Range. Due to limited battery range and insufficient charging infrastructure, the travel demand of BEV users is likely under constraint. BEV users may check the remaining SOC before each travel. Once the travel distance is beyond the remaining range, drivers have to charge the battery or change the travel plan. This situation is referred to as "range limitation" [15]. The more times the mileage limit occurs, the less user satisfaction will be.

We reconstruct the daily trip chain for BEV users by simulation. The remaining SOC is determined by the energy consumed in the previous travels and the availability of charging opportunity. Once there is no charging opportunity, the situation of "range limitation" may occur when the remaining battery range is not long enough for the next trip. The trip chain must be terminated as the battery may run out. We count the number of the days with incomplete trip chain and denote it as $\tau(R)$, where $R$ represents driving range. It can be speculated that the larger the battery, the less possibility that the range limitation occurs.

The fitness of driving range $\theta$ is defined as

$$
\theta=\left(1-\frac{\tau(R)}{M} \times 100 \%\right),
$$

where $M$ is the number of travel days for BEV users. The proposed battery fitness measure can incorporate stochastic driver behaviors by incorporating randomly distributed variables in daily trip chain. If the simulated travel days for a BEV are 10,000 , the fitness $\theta=95 \%$ means that there are 50 days when the BEV cannot fulfill the traveler's travel demand.

\section{Methodology}

\subsection{Modeling of Driver's Behaviors}

4.1.1. Data Description. This study makes use of a rich database collected from 50 BEVs over a period of 4-12 months (spanning from June 5, 2015, to June 30, 2016). The dataset is provided by Shanghai Electric Vehicles Data Center (SHEVDC) that is developed to remotely monitor electric vehicles driven across the city. The 50 BEVs, used as personal vehicles, are with the same model of Roewe E50, which is a pure electric passenger car with a $22.4 \mathrm{~kW} \cdot \mathrm{h}$ battery pack and a claimed driving range of $170 \mathrm{~km}$ under NEDC conditions [25].

Vehicle terminals, such as global positioning system data loggers and instruments to measure voltage and current, are installed on BEVs for data collection [26, 27]. The collected data includes turn on time, turn off time, total mileage, SOC, voltage, and current. Vehicles' driving information includes time-stamped location (i.e., longitude and latitude), spot speed, and azimuth. After data cleaning and consistency check procedure to remove invalid data and possible errors from the record, BEV owners' driving patterns, such as the trip distance, average travel speed, departure time of each trip, trip time length, SOC before/after each trip, and energy consumed, are extracted.

After the data cleaning of the original dataset, 12,855 valid trips, 7,112 accurate charges, and 7,275 travel days with authentic trips were extracted. Due to the intermittent record, the travel distance between two consecutive charges got 6,244 records. Table 2 summarizes the descriptive statistics of the valid trips and charges.

In terms of travel demand, the indicator that best reflects daily travel demand is the daily travel distance. Its average is $51.6 \mathrm{~km}$, which is only $30 \%$ of the battery's driving range. In addition, the average number of daily trips is 2.97 , and the highest value is 9 , which can be roughly understood as a daily commute user. For charging habits, the average SOC before charging is $45.6 \%$. This means that the user prefers to start connecting the charging plug when there is still about half of SOC remaining. 
TABLE 2: Summary metrics of valid trips and charges.

\begin{tabular}{|c|c|c|c|c|c|c|}
\hline & Number of samples & Avg. & Med. & Max. & Min. & St.D. \\
\hline Number of daily trips $N$ & 7275 & 2.97 & 2 & 9 & 1 & 1.16 \\
\hline The departure time for the first trip $T_{0}$ & 7275 & $11: 23$ & $10: 45$ & $23: 59$ & $0: 00$ & $4: 50$ \\
\hline Daily vehicle kilometers traveled $D(\mathrm{~km})$ & 7275 & 51.6 & 55.4 & 95.3 & 17.5 & 18.8 \\
\hline Travel time $t_{n}(\mathrm{~h})$ & 12855 & 0.71 & 0.57 & 9.12 & 0.05 & 0.54 \\
\hline Travel speed $v_{n}(\mathrm{~km} / \mathrm{h})$ & 12855 & 19.62 & 17.04 & 80 & 0.25 & 11.42 \\
\hline Dwell time between two consecutive trips $s_{n}(\mathrm{~h})$ & 6244 & 2.7 & 1.69 & 22.15 & 0.06 & 2.86 \\
\hline Charging power & 7112 & 3.80 & 3.21 & 6.71 & 1.31 & 2.64 \\
\hline SOC before charging $\operatorname{SOC}_{n}^{\prime}(\%)$ & 7112 & 45.6 & 45.4 & 61.6 & 18.5 & 8.3 \\
\hline
\end{tabular}

4.1.2. Modeling Variables for Daily Trips. In order to verify if there is dependence among the variables for daily trips, Pearson's linear correlation which measures a monotonic relationship between variables is estimated and placed in the matrix, $R$ [28]. Pearson's linear correlation derived for the variables representing daily trips: the departure time for the first trip $T_{0}$, daily vehicle kilometers traveled $D$, and number of daily trips $N$ are given by

$$
R=\left[\begin{array}{ccc}
1 & -0.23 & 0.51 \\
-0.23 & 1 & -0.35 \\
0.51 & -0.35 & 1
\end{array}\right]
$$

Testing the hypothesis of no correlation against the alternative that there is a nonzero correlation was applied to the elements of the correlation matrices [29]. All coefficients in the $R$ matrix are found to be statistically significant assuming a 0.05 level of significance. In the $R$ matrix, there is strong evidence that all variables are correlated and it is important to model the dependence structure between the core variables. According to $R(1,2)$, daily travel distance is negatively correlated with the first departure time of the day, which means that the later the user travels the earliest day, the shorter the daily travel mileage is. Meanwhile, $R(1,3)$ shows that the more daily trips, the greater the distance traveled each day. Therefore, after demonstrating the dependencies between the simulated variables, a joint PDF using their respective marginal distributions and a copula function needs to be constructed.

By modeling using copula function, we get the joint distribution result between the three variables for daily trips. If the resulting modeling structure is correct, the original and simulated values of the corresponding variables should have the same distribution. This is studied using the quantilequantile (Q-Q) plot of the simulated and raw data. Figure 1 shows the Q-Q plot of the raw and simulated values of the variables (daily vehicle kilometers traveled). If the two distributions to be compared are similar, the points in the Q-Q diagram will approximate a straight line. It can be seen from the figure that it approximates a straight line, indicating that the simulated and original data are from the same distribution.

\subsubsection{Modeling Variables for Each Trip}

(1) Travel Time $t_{n}$. Figure 2 plots the distribution of travel time for each trip. $54.28 \%$ of the travel time is less than 40 minutes. We find that the travel time $t_{n}$ follows the lognormal distribution [22]. The PDF of $t_{n}$ is written as

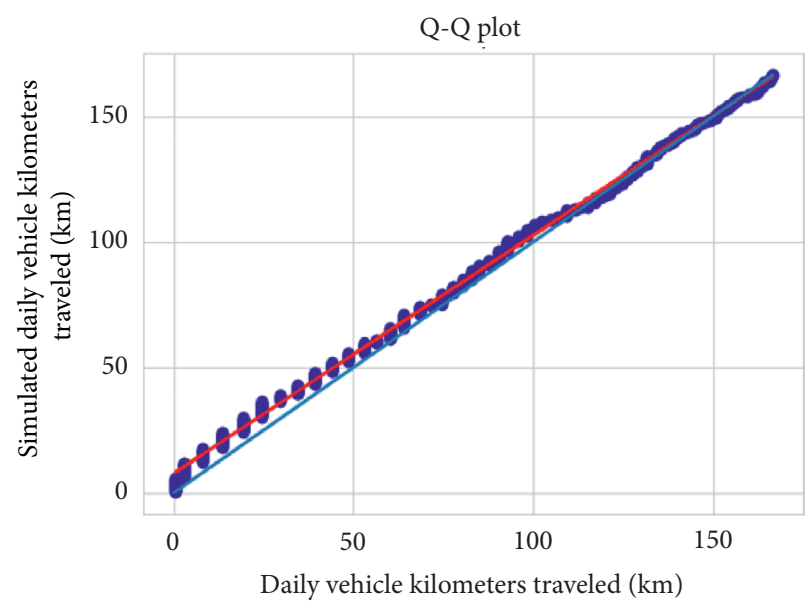

FIgURE 1: Q-Q plot of the simulated and original variable (daily vehicle kilometers traveled).

$$
f\left(t_{n}\right)=\frac{1}{\sqrt{2 \pi} \sigma t_{n}} \exp \left[-\frac{1}{2 \sigma^{2}}\left(\ln t_{n}-\mu\right)^{2}\right], \quad t_{n}>0,
$$

where $\mu=2.9115$ and $\sigma=0.876$.

(2) Travel Speed $v_{n}$. The travel speed for each trip follows the beta distribution, with an average value of $22.04 \mathrm{~km} / \mathrm{h}$ (Figure 3 ). The PDF of travel speed $v_{n}$ is expressed as

$$
\begin{aligned}
p_{3}(y) & =\frac{\Gamma(\alpha+\beta)}{\Gamma(\alpha)+\Gamma(\beta)} y^{\alpha-1}(1-y)^{\beta-1} . \\
y & =\frac{v_{n}-a}{b}
\end{aligned}
$$

where $\alpha=2.9339, \beta=3256600, a=0.50467$, and $b=2399300$.

(3) Dwell Time between Two Consecutive Trips $s_{n}$. Once a trip is ended, BEV drivers may stop at the destination for a certain period. The dwell time is one of the key factors for charging decision [30]. Figure 4 depicts the distribution of dwell time between two consecutive trips. $57.5 \%$ of the dwells last for less than 2 hours, which may not be appropriate for charging with slow charging. The dwell time is estimated by a lognormal distribution. The PDF of $s_{n}$ is written as

$$
p_{4}\left(s_{n}\right)=\frac{1}{\sqrt{2 \pi} \sigma s_{n}} \exp \left[-\frac{1}{2 \sigma^{2}}\left(\ln s_{n}-\mu\right)^{2}\right], \quad x>0,
$$

where $\mu=2.6$ and $\sigma=1.0147$. 


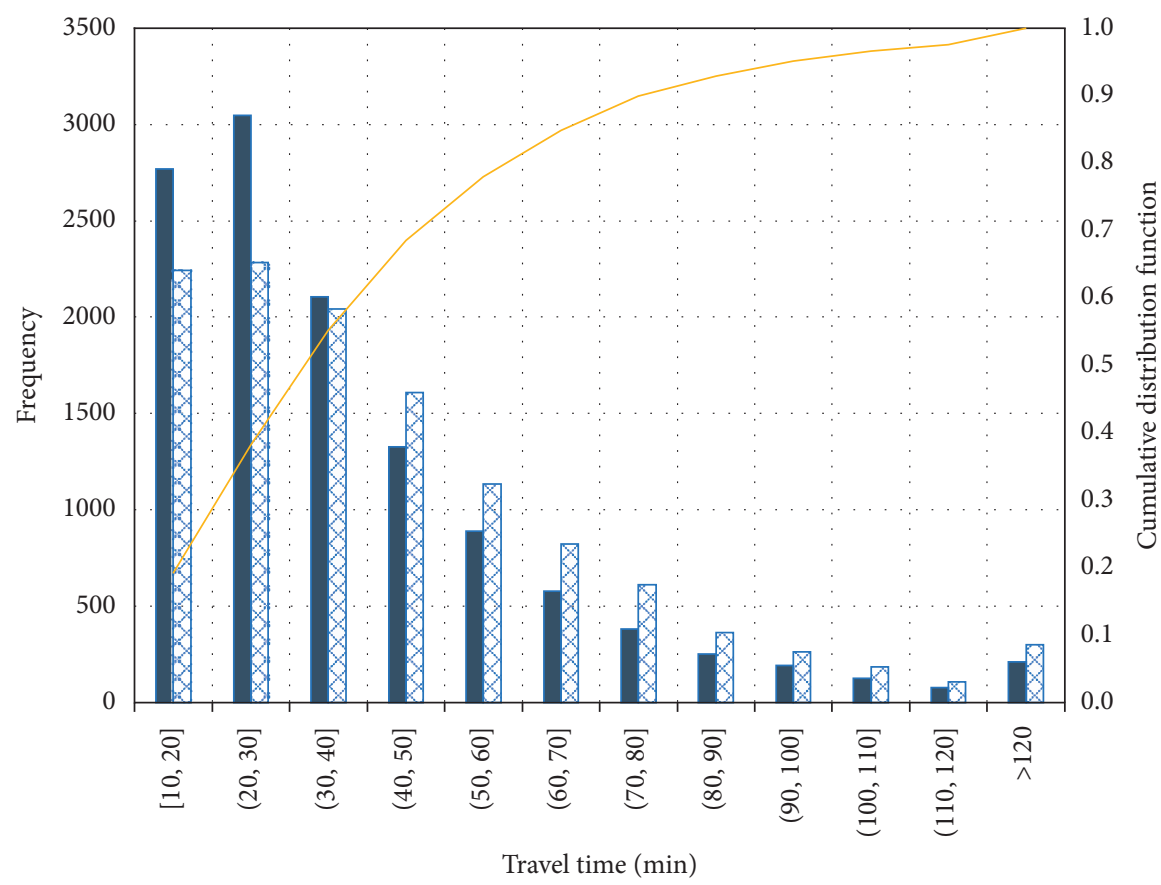

Original data

ॠख Fitted value

CDF

Figure 2: The distribution of travel time.

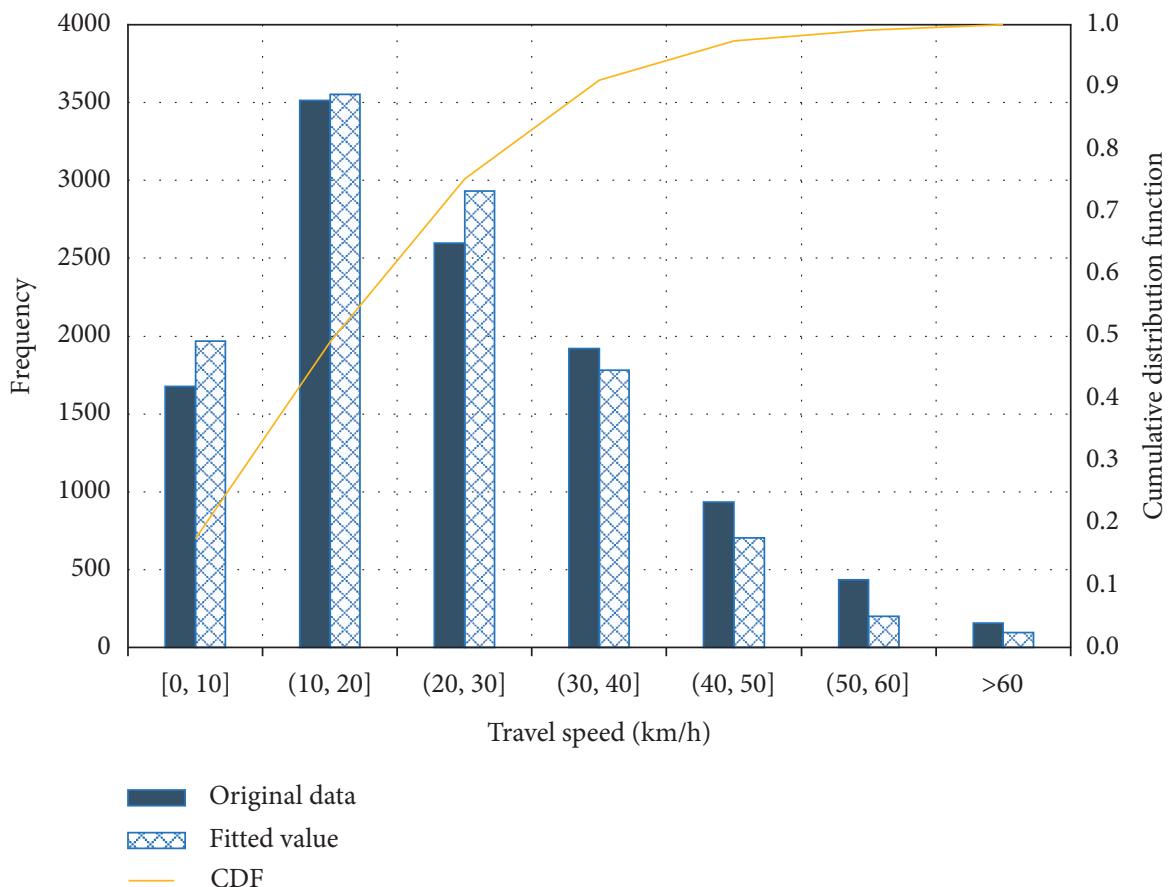

Figure 3: The distribution of travel speed.

(4) SOC before Charging, SOC ${ }_{n}^{\prime}$. The SOC before charging is adopted as the threshold for each charging event. Once the remaining SOC is above the threshold, the charging event will not occur [31]. As shown in Figure 5, the preference of the
SOC before charging is not obvious, indicating drivers are likely to charge their vehicles whenever they have charging opportunities. The distribution of $\mathrm{SOC}_{n}^{\prime}$ is found subject to the Johnson SB distribution. The PDF of $\mathrm{SOC}_{n}^{\prime}$ is written as 


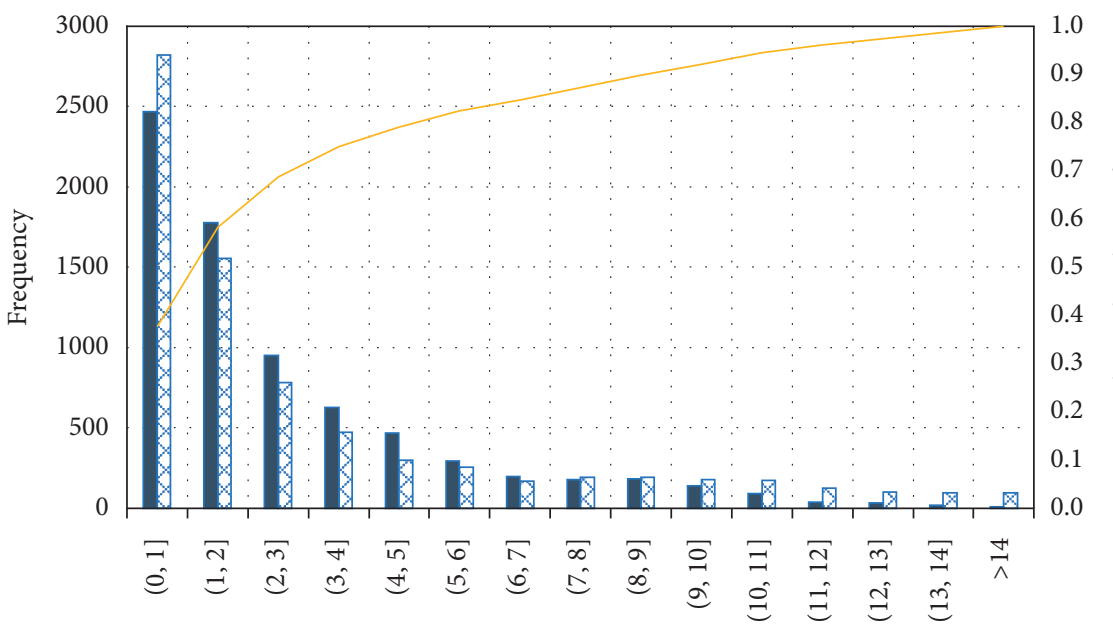

Dwell time between two consecutive trips (h)

Original data

$\bowtie$ Fitted value

$\mathrm{CDF}$

Figure 4: The distribution of dwell time between two consecutive trips.

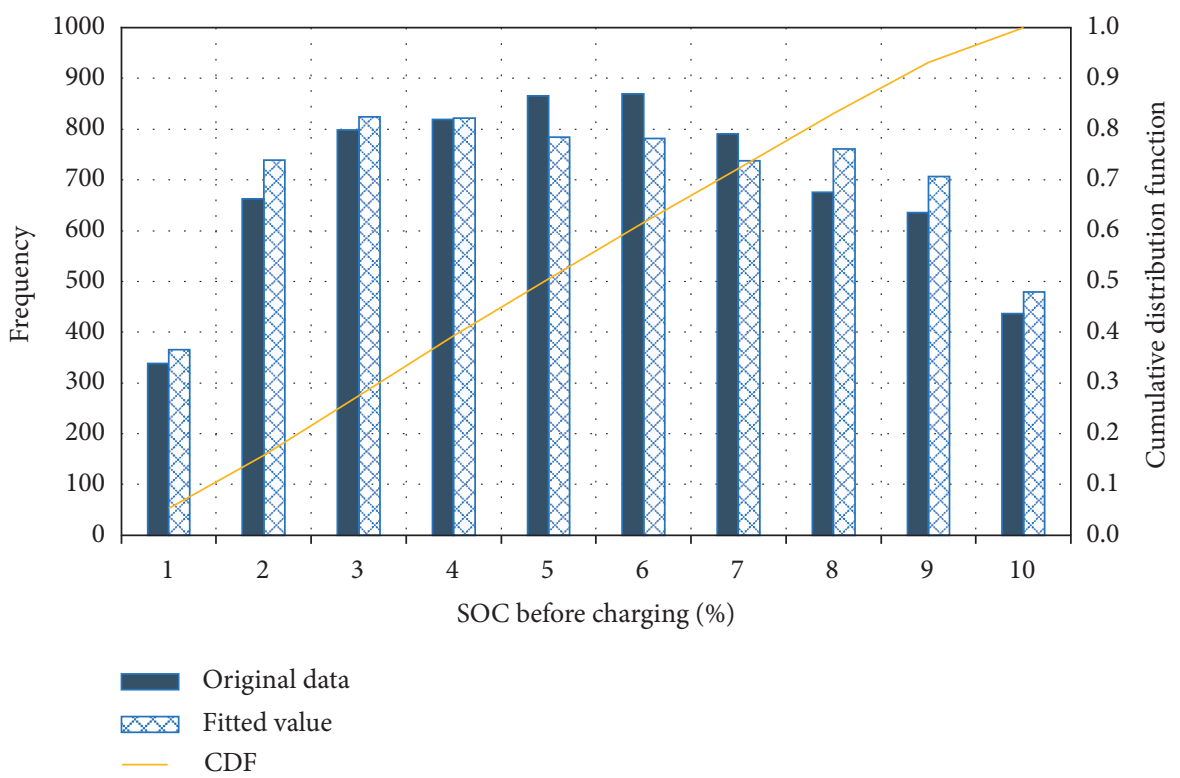

Figure 5: The distribution of SOC before charging.

$$
\begin{aligned}
p_{5}\left(t_{n}\right) & =\frac{\delta}{\sqrt{2 \pi} z(1-z)} \exp \left(-\frac{1}{2}\left(\gamma+\delta \ln \left(\frac{z}{1-z}\right)\right)^{2}\right), \quad \zeta \leq z \leq \zeta+\lambda, \\
z & =\frac{\operatorname{SOC}_{n}^{\prime}-\lambda}{\lambda}
\end{aligned}
$$

where $\gamma=0.081, \delta=0.242, \zeta=0$, and $\lambda=100$.

4.1.4. Drivers Heterogeneity. In order to consider the impact of heterogeneity, Yang et al. [11] applied a machine learning method to analyze BEV drivers' habitual behaviors based on the same sample data. User heterogeneity is mainly reflected in different travel patterns. Daily vehicle kilometers traveled $D$ are not only the core of the behavior variable for daily trips, but also the dependency of each trip variables. Therefore, the research on user heterogeneity in this paper mainly focuses on the optimal driving range of different daily travel users. According to the results of Yang et al. [11], the $50 \mathrm{BEV}$ sample vehicles can be divided into 4 clusters in Table 3 by daily vehicle kilometers traveled $D$. Obviously, based on the same charging scenario restrictions, users with different travel pattern will have corresponding optimal driving range. 
TABLe 3: Summary metrics of different clusters.

\begin{tabular}{lcccc}
\hline \multirow{2}{*}{ Type } & Number of vehicles & \multicolumn{3}{c}{$\begin{array}{c}\text { Avg. daily vehicle kilometers } \\
\text { traveled (km) }\end{array}$} \\
& & Sample size & Mean & St.D. \\
\hline Cluster A & 34 & 4406 & 58.0 & 42.0 \\
Cluster B & 2 & 240 & 93.4 & 44.3 \\
Cluster C & 5 & 1059 & 31.7 & 30.1 \\
Cluster D & 9 & 1570 & 23.3 & 19.0 \\
\hline
\end{tabular}

\subsection{Monte Carlo Simulation}

4.2.1. Simulation Assumptions. The data set of this study is based on the Roewe E50, so the initial driving range $R$ during the simulation is also $170 \mathrm{~km}$. That is, the driving range limitation of this study is specifically defined for this vehicle model. Several conditions are set for the simulation context/ environment. They are summarized as follows.

Condition 1. Charging places: two scenarios are set for charging locations. In Scenario 1, users can only charge the battery at home when they go back home after the last trip of the travel day. In Scenario 2, the charging facility is available at workplace, and users can choose to charge at the workplace or charge at home. Two time periods for workplace charging are set, i.e., from 09:00 to 16:00 [32].

Condition 2. Energy consumption: the electricity consumed for the $n$th trip $E_{n}^{t}(\mathrm{~kW})$ is supposed to be determined by the travel time and travel distance [33]. A linear relationship is found based on the real-world data with the $R$-squared value of 0.937. It can be written as

$$
E_{n}^{t}=0.0038 t_{n}+0.8358 l_{n}+0.9829,
$$

where $l_{n}(\mathrm{~km})$ is travel distance for the $n$th trip determined by $v_{n}$ and $t_{n}$.

Condition 3. Charged energy: the amount of electricity charged mainly depends on the charging power and the dwell time. The charging power is assumed as constant, and the charged electricity $E_{n}^{c}(\mathrm{~kW})$ for the $n$th dwell can be written as

$$
E_{n}^{c}=\min \left(R_{n}^{r}+p \times s_{n}, R\right),
$$

where $R_{n}^{r}(\mathrm{~kW} \cdot \mathrm{h})$ is the remaining range after the $n$th trip, $p$ is the charging power, and $R(\mathrm{~kW} \cdot \mathrm{h})$ is the battery capacity.

4.2.2. Simulation Process. Based on the distribution models of drivers' driving and charging behaviors, the Monte Carlo simulation is applied to simulate the trip chain for BEV users. To stabilize the simulation results, the number of the simulated travel days $M$ is set as 10,000 . The simulation process is presented in Algorithm 1.

\section{Results and Discussion}

The charging problem is a key obstacle hindering the development of BEVs. The feasible methods to solve the charging problem mainly include increasing the charging opportunity and increasing the power of the charging pile. These two feasible methods will be studied to influence the optimal driving range of different users in this section.

5.1. Impact of Workplace Charging. In general, household charging piles mainly apply alternating current (AC), which is mainly divided into two levels. Level 1 operates at 120 VAC (voltage in an alternating current), while Level 2 uses 208 or $240 \mathrm{VAC}$, and the corresponding charging power ranges from $1.4 \mathrm{~kW}$ to $7.2 \mathrm{~kW}$. If the charging pile in public places uses direct current (DC), the charging power can reach $50 \mathrm{~kW}$ or more. Although the charging power of Electric Vehicle Supply Equipment (EVSE) is now getting bigger, even more than $100 \mathrm{~kW}$, it takes a long time to spread to the average drivers and to apply to each $\mathrm{BEV}$ model $[34,35]$. Almost all charging events extracted from the dataset are performed using AC Level 2 Electric Vehicle Supply Equipment (EVSE), with an average value of $3.8 \mathrm{~kW}$ in Table 2. Thus, by setting three kinds of slow charging power (i.e., the power of $3.5 \mathrm{~kW}, 7 \mathrm{~kW}$, and $15 \mathrm{~kW}$ ) at two charging places, the impact of increasing the charging opportunity on the optimal driving range is studied. Given the fitness requirement $\theta=95 \%$, Figure 6 presents the results of optimal driving range in Scenario 1 and Scenario 2.

Whether it is to increase the charging opportunity or the charging power, the value of the optimal driving range can be reduced, because the daily travel demand of users is more easily met. In detail, the daily vehicle kilometers traveled of clusters $A$ and $B$ exceed the average value in Table 2 . Compared with Scenario 1, Scenario 2 that increases the charging opportunity at the workplace has a significant effect on reducing the optimal driving range, especially for cluster $B$ with a daily vehicle kilometers traveled value of $93 \mathrm{~km}$. Clusters $C$ and $D$ with smaller travel demand are not sensitive enough to Scenario 1 and 2 with different charging opportunities. When the charging power is $15 \mathrm{~kW}$, the optimal driving range of both clusters is only slightly reduced.

According to the original driving range of the Roewe E50 $(170 \mathrm{~km})$, two drivers in cluster $B$ are not suitable for this model unless the charging power and charging opportunities are appropriately increased. Also, the daily travel demand of the 14 users in clusters $C$ and $D$ can be mostly satisfied even if charging only at home and the charging power is $3.5 \mathrm{~kW}$. As the daily vehicle kilometers traveled of cluster $A$ are similar to the average value, it can be found that when the average daily travel distance of the user accounts for about 30\%-34\% of the battery driving range, the fitness of battery capacity cannot be satisfied. Therefore, users should choose their own BEVs whose daily travel demand is within $34 \%$ of the battery driving range.

5.2. Impacts of Fast Charging. In order to understand the impact of fast charging, based on the results of Section 5.1, the benchmark scenario is set to $15 \mathrm{~kW}$ at home and at the workplace. Assuming fast charging is only available at workplace, the added charging power of workplaces (CPW) 
Input: Practical data of the behavioral variables of the daily trip chain in Table 1

Output: Simulated travel and charging process

Process:

Initial: $M=10,000, m=1, i=0$.

Step 0: the vehicle is assumed to depart from home with a full battery

Step 1: generate the daily trip, the number of daily trips $N$, the departure time for the first trip $T_{0}$, and the daily travel distance $D$ using copula function; set $i=1$

Step 2: generate the travel distance, the average travel speed for each trip, and the dwell time between the two trips by equation (2) on the day $m$

Step 3: calculate the electricity consumed for the $i^{\text {th }}$ trip; check whether the remaining SOC is sufficient for the $i^{\text {th }}$ trip. If it is, update the remaining SOC after arriving at the destination, and proceed to Step 4; otherwise, the $i^{\text {th }}$ trip is marked as failed with "range limitation," and choose alternative travel mode to make up for this trip; set $m=m+1$, and return to Step 0

Step 4: generate SOC before charging SOC $_{n}^{\prime}$; check whether a charging opportunity is available at that time. If the charge is performed, calculate the increased SOC and update the remaining range; otherwise, the remaining range keeps the same

Step 5: check whether $i$ equals $N$. If it does, move to Step 6; otherwise, $i=i+1$, and return to Step 2

Step 6: check whether $m$ equals $M$. If it does, the simulation is terminated; otherwise, set $m=m+1$, and return to Step 1

Algorithm 1: Summary of simulation methodology.

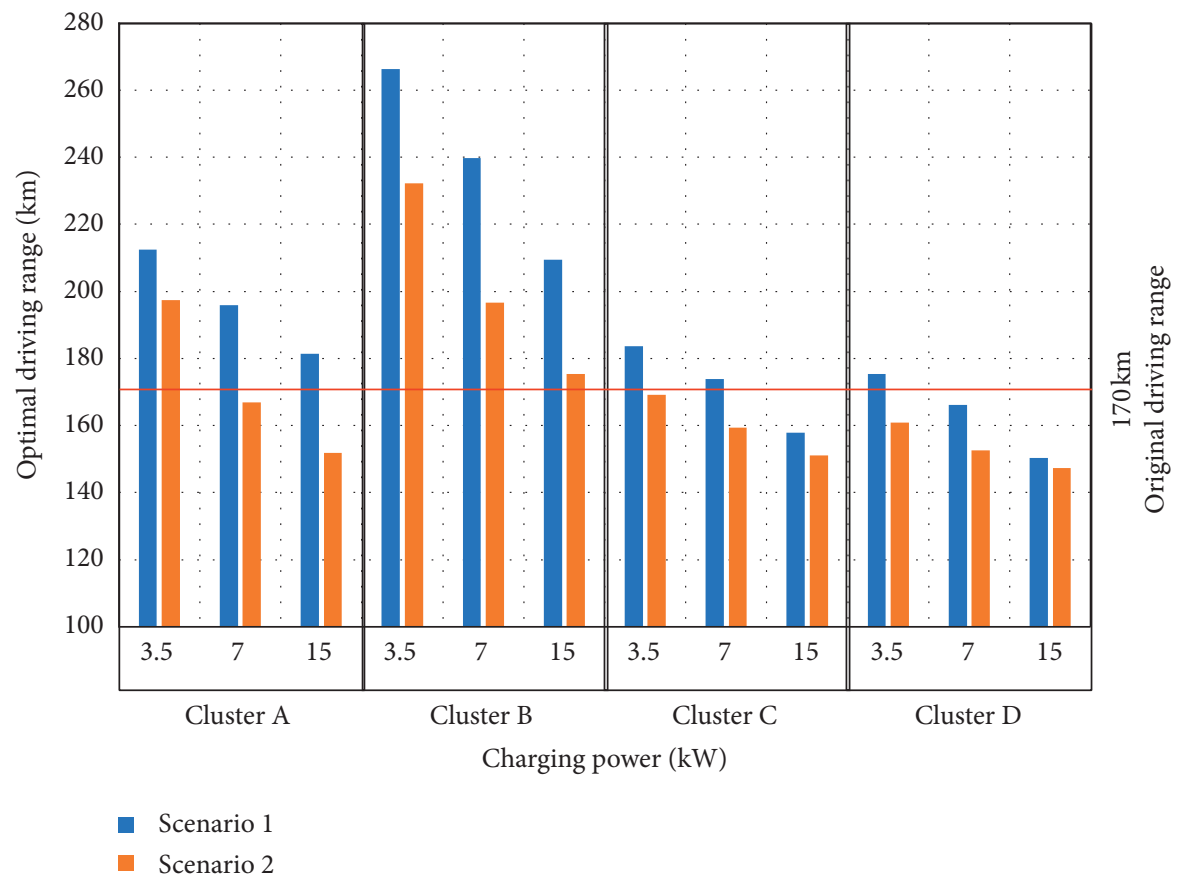

FIgURe 6: Optimal driving range for two scenarios when $\theta=95 \%$.

is set to $30 \mathrm{~kW}, 45 \mathrm{~kW}$, and $50 \mathrm{~kW}$, respectively. The charging power at home $(\mathrm{CPH})$ is still set to $15 \mathrm{~kW}$. Figure 7 shows a stacked bar chart of relative reduction percentages when the optimal driving range is compared with the values of the benchmark scenario as CPW increases.

Cluster B, which has the largest daily vehicle kilometers traveled, has the largest reduction percentage. When $\mathrm{CPW}=50 \mathrm{~kW}$ and $\mathrm{CPH}=15 \mathrm{~kW}$, the optimal driving range can be reduced by $10.4 \%$ compared with the benchmark scenario. Conversely, the cumulative reduction percentage of clusters $C$ and $D$ is in the range of $7.6 \%-8.4 \%$. The benefits of fast charging are more significant to users with high daily travel demand. Besides, as the CPW increases, the value of the relative reduction percentage for each cluster gradually decreases. It can be seen that, on the premise of meeting travel demand, the beneficial effects brought by the increase in fast-charging power will gradually weaken.

To sum up, with the increase in daily travel demand of residents, the beneficial effects of charging piles equipped with fast-charging power can be reflected. The government should pay more attention to the layout of the fast-charging piles in the area where residents with large daily travel needs are located. Simultaneously, a reasonable amount of fastcharging power should be studied without the need for blind high power. 


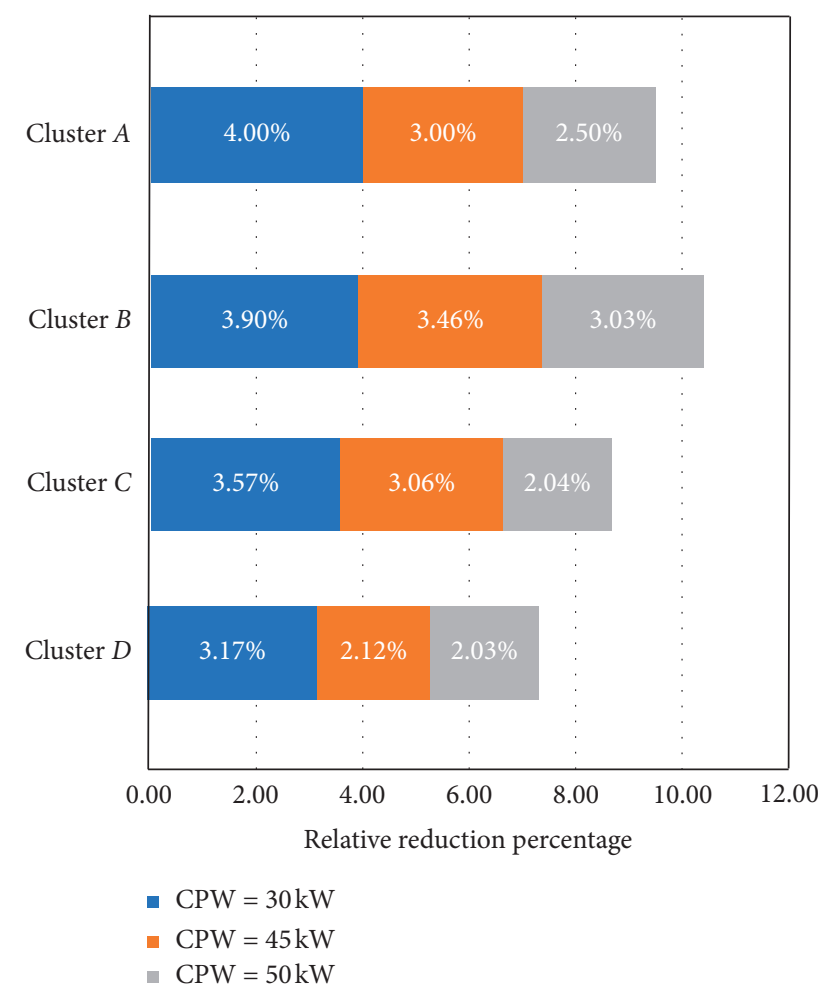

FIGURE 7: A stacked bar chart of relative reduction percentages.

\section{Conclusions}

Aimed at finding out the optimal driving range considering user heterogeneity in different charging scenarios, this study proposes a simulation modeling method representing BEV users' driving and charging behavior using the real-world in-use data collected from Shanghai, China. The Monte Carlo simulation is adopted to reconstruct BEV users' daily trip chains and quantify the fitness of the driving range. The key findings from the results include the following. (1) The daily travel chain can be reconstructed by variables for daily trips and each trip, and dependencies exist in these variables. (2) According to the results of four clusters, users with different daily travel demand have different optimal driving ranges. When choosing their BEV, users should consider that the daily vehicle kilometers traveled are less than $34 \%$ of the battery driving range. (3) Increasing both the charging opportunity and charging power is more beneficial to drivers with large daily vehicle kilometers traveled. (4) With the increase of fast-charging power, this beneficial effect gradually weakens when daily travel demand is met.

The main contribution of this paper is to construct a daily trip chain to analyze the optimal driving ranges of various heterogeneous users. However, due to errors in the vehicle's latitude and longitude in the data source, the restriction is that only the home and workplace are assumed during the daily trip chain. The purpose of daily activities of residents is diverse, not only work and home. Therefore, further enriching the daily trip chain is also the main focus in the future research direction.

\section{Data Availability}

The data used to support the findings of this study have not been made available because of confidential issues.

\section{Conflicts of Interest}

The authors declare that there are no conflicts of interest regarding the publication of this article.

\section{Acknowledgments}

This study was supported by the National Key Research and Development Program of China (No. 2018YFB1600900) and the National Natural Science Foundation of China (No. 71971060). The analysis data provided by Shanghai International Automobile City (SIAC) supports this study.

\section{References}

[1] R. R. Desai, R. B. Chen, and W. Armington, "A pattern analysis of daily electric vehicle charging profiles: operational efficiency and environmental impacts," Journal of Advanced Transportation, vol. 2018, Article ID 6930932, 15 pages, 2018.

[2] A. D. Alvarez, F. S. Garcia, J. E. Naranjo, J. J. Anaya, and F. Jimenez, "Modeling the driving behavior of electric vehicles using smartphones and neural networks," IEEE Intelligent Transportation Systems Magazine, vol. 6, no. 3, pp. 44-53, 2014.

[3] Y. Guo, Z. Li, Y. Wu, and C. Xu, "Evaluating factors affecting electric bike users' registration of license plate in China using bayesian approach," Transportation Research Part F: Traffic Psychology and Behaviour, vol. 59, pp. 212-221, 2018.

[4] K. Hu, J. Wu, and T. Schwanen, "Differences in energy consumption in electric vehicles: an exploratory real-world study in Beijing," Journal of Advanced Transportation, vol. 2017, Article ID 4695975, 17 pages, 2017.

[5] N. S. Pearre, W. Kempton, R. L. Guensler, and V. V. Elango, "Electric vehicles: how much range is required for a day's driving?" Transportation Research Part C: Emerging Technologies, vol. 19, no. 6, pp. 1171-1184, 2011.

[6] M. Helmbrecht, C. Olaverri-Monreal, K. Bengler, R. Vilimek, and A. Keinath, "How electric vehicles affect driving behavioral patterns," IEEE Intelligent Transportation Systems Magazine, vol. 6, no. 3, pp. 22-32, 2014.

[7] A. Dimitropoulos, P. Rietveld, and J. N. V. Ommeren, "Consumer valuation of changes in driving range: a metaanalysis," Transportation Research Part A: Policy and Practice, vol. 55, pp. 27-45, 2011.

[8] T. Miwa, H. Sato, and T. Morikawa, "Range and battery depletion concerns with electric vehicles," Journal of Advanced Transportation, vol. 2017, Article ID 7491234, 12 pages, 2017.

[9] X. Wu, D. Freese, A. Cabrera, and W. A. Kitch, "Electric vehicles' energy consumption measurement and estimation," Transportation Research Part D: Transport and Environment, vol. 34, no. 34, pp. 52-67, 2015.

[10] Z. Lin, J. Dong, C. Liu, and D. greene, "Estimation of energy use by plug-in hybrid electric vehicles," Transportation Research Record: Journal of the Transportation Research Board, vol. 2287, no. 1, pp. 37-43, 2012.

[11] J. Yang, J. Dong, Q. Zhang, Z. Liu, and W. Wang, "An investigation of battery electric vehicle driving and charging 
behaviors using vehicle usage data collected in Shanghai, China," Transportation Research Record Journal of the Transportation Research Board, vol. 2672, 2018.

[12] W. Jianfeng, T. Shun, X. Xiangning, L. Chen, and L. Kunyu, "Analysis on charging demand of ev based on stochastic simulation of trip chain," Power System Technology, vol. 39, no. No. 6, pp. 1477-1485, 2015.

[13] Y. Liu, Z. Liu, and R. Jia, "DeepPF: a deep learning based architecture for metro passenger flow prediction," Transportation Research Part C: Emerging Technologies, vol. 101, pp. 18-34, 2019.

[14] T. Franke and J. F. Krems, "What drives range preferences in electric vehicle users?" Transport Policy, vol. 30, no. 3, pp. 56-62, 2013.

[15] J. Dong and Z. Lin, "Stochastic modeling of battery electric vehicle driver behavior," Transportation Research Record: Journal of the Transportation Research Board, vol. 2454, no. 1, pp. 61-67, 2014.

[16] Q. Cheng, Z. Liu, and W. Y. Szeto, “A cell-based dynamic congestion pricing scheme considering travel distance and time delay," Transportmetrica B: Transport Dynamics, vol. 7, no. 1, pp. 1286-1304, 2019.

[17] J. Bao, P. Liu, X. Qin, and H. Zhou, "Understanding the effects of trip patterns on spatially aggregated crashes with large-scale taxi GPS data," Accident Analysis \& Prevention, vol. 120, pp. 281-294, 2018.

[18] A. S. Al-Ogaili, T. J. Tengku Hashim, N. A. Rahmat et al., "Review on scheduling, clustering, and forecasting strategies for controlling electric vehicle charging: challenges and recommendations," IEEE Access, vol. 7, pp. 128353-128371, 2019.

[19] Z. Li, S. Jiang, J. Dong, S. Wang, Z. Ming, and L. Li, “Battery capacity design for electric vehicles considering the diversity of daily vehicles miles traveled," Transportation Research Part C: Emerging Technologies, vol. 72, pp. 272-282, 2016.

[20] J. Brady and M. O’Mahony, "Modelling charging profiles of electric vehicles based on real-world electric vehicle charging data," Sustainable Cities and Society, vol. 26, pp. 203-216, 2016.

[21] Z. Shuqiang, Z. Jingren, L. Zhiwei, and Z. Shuo, "EV charging demand analysis based on trip chain theory," Electric Power Automation Equipment, vol. 37, no. 8, pp. 105-117, 2017.

[22] E. Chen, Z. Ye, C. Wang, and M. Xu, "Subway passenger flow prediction for special events using smart card data," IEEE Transactions on Intelligent Transportation Systems, vol. 21, 2019.

[23] G. Papaefthymiou and D. Kurowicka, "Using copulas for modeling stochastic dependence in power system uncertainty analysis," IEEE Transactions on Power Systems, vol. 24, no. 1, pp. 40-49, 2008.

[24] M. Sklar, "Fonctions de repartition an dimensions et leurs marges," Publications from the Institute of Statistics of the University of Paris, vol. 8, pp. 229-231, 1959.

[25] ROEWE, The Introcution of Roewe E50, ROEWE, Shanghai, China, 2018, http://www.roewe.com.cn/roewee50/.

[26] X. Gu, M. Abdel-Aty, Q. Xiang, Q. Cai, and J. Yuan, "Utilizing UAV video data for in-depth analysis of drivers' crash risk at interchange merging areas," Accident Analysis \& Prevention, vol. 123, pp. 159-169, 2019.

[27] Y. Yuan, M. Yang, J. Wu, S. Rasouli, and D. Lei, “Assessing bus transit service from the perspective of elderly passengers in Harbin, China," International Journal of Sustainable Transportation, vol. 13, pp. 1-16, 2019.

[28] C. Wang, C. Xu, J. Xia, Z. Qian, and L. J. T. r. p. C. e. t. Lu, “A combined use of microscopic traffic simulation and extreme value methods for traffic safety evaluation," Transportation Research Part C: Emerging Technologies, vol. 90, pp. 281-291, 2018.

[29] D. Kurowicka and R. M. Cooke, Uncertainty Analysis with High Dimensional Dependence Modelling, John Wiley \& Sons, Hoboken, NJ, USA, 2006.

[30] L. Li, J. Zhang, Y. Wang, and B. Ran, "Missing value imputation for traffic-related time series data based on a multiview learning method," IEEE Transactions on Intelligent Transportation Systems, vol. 20, 2018.

[31] T. Franke, M. Günther, M. Trantow, and J. F. Krems, "Does this range suit me? Range satisfaction of battery electric vehicle users," Applied Ergonomics, vol. 65, pp. 191-199, 2017.

[32] V. Torres-Sanz, J. A. Sanguesa, F. J. Martinez, P. Garrido, and J. M. Marquez-Barja, "Enhancing the charging process of electric vehicles at residential homes," IEEE Access, vol. 6, pp. 22875-22888, 2018.

[33] Y. Pan, S. Chen, F. Qiao, S. V. Ukkusuri, and K. Tang, "Estimation of real-driving emissions for buses fueled with liquefied natural gas based on gradient boosted regression trees," Science of the Total Environment, vol. 660, pp. 741-750, 2019.

[34] H. Wang, D. Zhao, Q. Meng, G. P. Ong, and D.-H. Lee, "A four-step method for electric-vehicle charging facility deployment in a dense city: an empirical study in Singapore," Transportation Research Part A: Policy and Practice, vol. 119, pp. 224-237, 2019.

[35] A. Dubey and S. Santoso, "Electric vehicle charging on residential distribution systems: impacts and mitigations," IEEE Access, vol. 3, pp. 1871-1893, 2015. 